\title{
Relative validity of fruit and vegetable intake estimated from an FFQ, using carotenoid and flavonoid biomarkers and the method of triads
}

\author{
Monica H. Carlsen ${ }^{1 *}$, Anette Karlsen ${ }^{1}$, Inger T. L. Lillegaard ${ }^{1}$, Jon M. Gran ${ }^{2}$, Christian A. Drevon ${ }^{1}$, \\ Rune Blomhoff ${ }^{1}$ and Lene F. Andersen ${ }^{1}$ \\ ${ }^{1}$ Department of Nutrition, Institute of Basic Medical Sciences, University of Oslo, Oslo, Norway \\ ${ }^{2}$ Department of Biostatistics, Institute of Basic Medical Sciences, University of Oslo, Oslo, Norway
}

(Received 31 March 2010 - Revised 15 November 2010 - Accepted 16 November 2010 - First published online 28 January 2011)

\begin{abstract}
The aim of the present study was to validate the intakes of fruit, juice and vegetables from an FFQ. In sub-study I ( $n$ 147), intakes from the FFQ were evaluated against $7 \mathrm{~d}$ weighed food records (WR) and plasma carotenoid concentrations, whereas in sub-study II ( $n$ 85), the intakes were evaluated against plasma carotenoid concentrations and amounts of flavonoids in $24 \mathrm{~h}$ urine samples. Relative validity was evaluated by comparing median intakes, estimating correlation coefficients and validity coefficients using the method of triads. In substudy I, we observed no significant difference in daily median fruit intake between the FFQ and the WR, whereas the intake of vegetables was higher from the FFQ than from the WR. The correlations between intakes from the FFQ and the WR ranged from $0 \cdot 31$ to $0 \cdot 58$. In sub-study II, the intakes of fruit and vegetables correlated significantly with plasma carotenoid concentrations and urinary flavonoids. The validity coefficients for the intakes of fruit and vegetables from the FFQ ranged from 0.61 to 0.88 in sub-study I and from 0.60 to 0.94 in sub-study II. In summary, based on the associations observed between intakes from the FFQ and the biomarkers and the FFQ validity coefficients, the FFQ was found valid and suitable for ranking individuals according to their usual intake of fruit, juice and vegetables.
\end{abstract}

Key words: FFQ: Plasma biomarkers: Urinary biomarkers: Method of triads: Fruit and vegetable intake

Over the last decades, nutritional research has provided data strongly suggesting beneficial health effects from a plantbased diet ${ }^{(1-9)}$. A typical plant-based diet provides thousands of phytochemicals ${ }^{(8)}$ that may modify a multitude of cellular processes $^{(10-13)}$. In our effort to elucidate the effects of dietary phytochemicals on health and disease, we have developed an FFQ for the assessment of phytochemical-rich food and beverages in a habitual diet. Assessment of long-term dietary intake using methods of self-reporting has generally been associated with a substantial degree of measurement errors $^{(14)}$. Self-reporting challenges the participants' memory and ability to take into account the variability in intake, from day to day or by season. Because of these obvious sources of errors, dietary assessment methods should always be validated before use. Dietary validation studies aim to estimate the association between dietary assessment data and the subjects' true intake ${ }^{(15)}$, and are usually performed comparing the test method with a more valid reference method. Measuring biomarkers of intake in biological samples is an objective method to assess dietary intake, although individual differences in digestion, absorption, distribution, metabolism and excretion should be taken into account ${ }^{(16)}$. Flavonoids and carotenoids are widely distributed phytochemicals in fruit and vegetables that are regularly consumed by humans. Several studies have investigated the use of plasma carotenoids and urinary flavonoids as biomarkers of fruit and vegetable intake ${ }^{(17-26)}$. Of the more than 700 natural carotenoids identified thus far, $\alpha$-carotene, $\beta$-carotene, $\beta$-cryptoxanthin, lutein, lycopene and zeaxanthin represent more than $95 \%$ of total blood carotenoids ${ }^{(27)}$. The type and amount of carotenoids differ among fruit and vegetables, thus different populations may have different plasma carotenoid profiles depending on what type of fruit and vegetables they consume ${ }^{(28)}$. The motivation for using urinary flavonoids as biomarkers came from earlier observations showing that the sum of flavonoids is associated with the consumption of fruit and vegetables $^{(18,20,22,26)}$. Urinary flavonoids reflect low ${ }^{(20)}$ and high intakes $^{(18,22)}$ as well as moderate changes in fruit and vegetable consumption $^{(18)}$.

Using more than one reference method in the validation study enabled us to apply the method of triads for evaluating the intakes of fruit and vegetables from the $\mathrm{FFQ}^{(15,29)}$.

Abbreviations: PABA, para-amino benzoic acid; VC, validity coefficient; WR, weighed food record.

*Corresponding author: M. H. Carlsen, fax +47 22851398, email m.h.carlsen@medisin.uio.no 
Moreover, measuring two biomarkers of intake made it possible to fulfil the assumption of two independent reference measures of intake ${ }^{(29)}$. The aim of the present study was thus to evaluate the relative validity of fruit, juice and vegetable intake from the FFQ, using the method of triads, first by applying data from weighed food records (WR) and plasma carotenoids and, second, by using urinary flavonoids and plasma carotenoids.

\section{Materials and methods}

\section{Subjects and study design}

The validation study was designed to include a representative sample of Norwegians aged 18-80 years. A total of 4500 randomly selected inhabitants of the Norwegian capital and surrounding area (administered by the National Tax Office/ Population Registration Office) received a written invitation to participate in the project. Exclusion criteria were pregnancy, weight loss of more than $5 \mathrm{~kg}$ during the last 6 months before the study and participation in drug testing trials. In total, 504 subjects responded to the invitation, and after a screening interview, 346 participants were enrolled in the study ( $8 \%$ participation rate). They received the FFQ and written instructions by mail and were asked to fill in the FFQ at home. Within 2 weeks, the participants attended a physical examination where overnight fasting blood samples were collected, the FFQ was returned and the participants were randomly assigned to three sub-studies: 168 participants were assigned to sub-study I and given instructions on how to carry out the $7 \mathrm{~d}$ WR; 114 participants were assigned to substudy II and asked to collect $24 \mathrm{~h}$ urine samples (Fig. 1). The remaining sixty-four participants were assigned to carry out an energy intake validation study. The results from the latter sub-study are published elsewhere ${ }^{(30)}$. The three parallel studies were designed to minimise the workload of the participants. Data collection was carried out from September 2006 until October 2007. The present study was conducted according to the guidelines laid down in the Declaration of Helsinki, and all procedures involving human subjects were approved by the Regional Ethics Committee for Medical Research. Written informed consent was obtained from all subjects.

\section{The semi-quantitative $F F Q$}

The fourteen-page questionnaire was designed to capture the habitual food intake among Norwegian adults during the preceding year. The FFQ was an extended and revised version of the semi-quantitative FFQ used in the Norwegian nationwide survey NORKOST 1997 (NFFQ) $^{(31)}$. The original NFFQ was a validated, 180-item optical readable FFQ, developed to cover total energy intake of the population ${ }^{(32-35)}$. Based on our extensive screening of antioxidant contents in food and beverages $^{(36)}$, the NFFQ was updated with questions about food products and food categories assumed to be important sources of dietary antioxidants in Norway. The new FFQ included 270 food items, grouped together according to the Norwegian meal pattern. The additional questions included

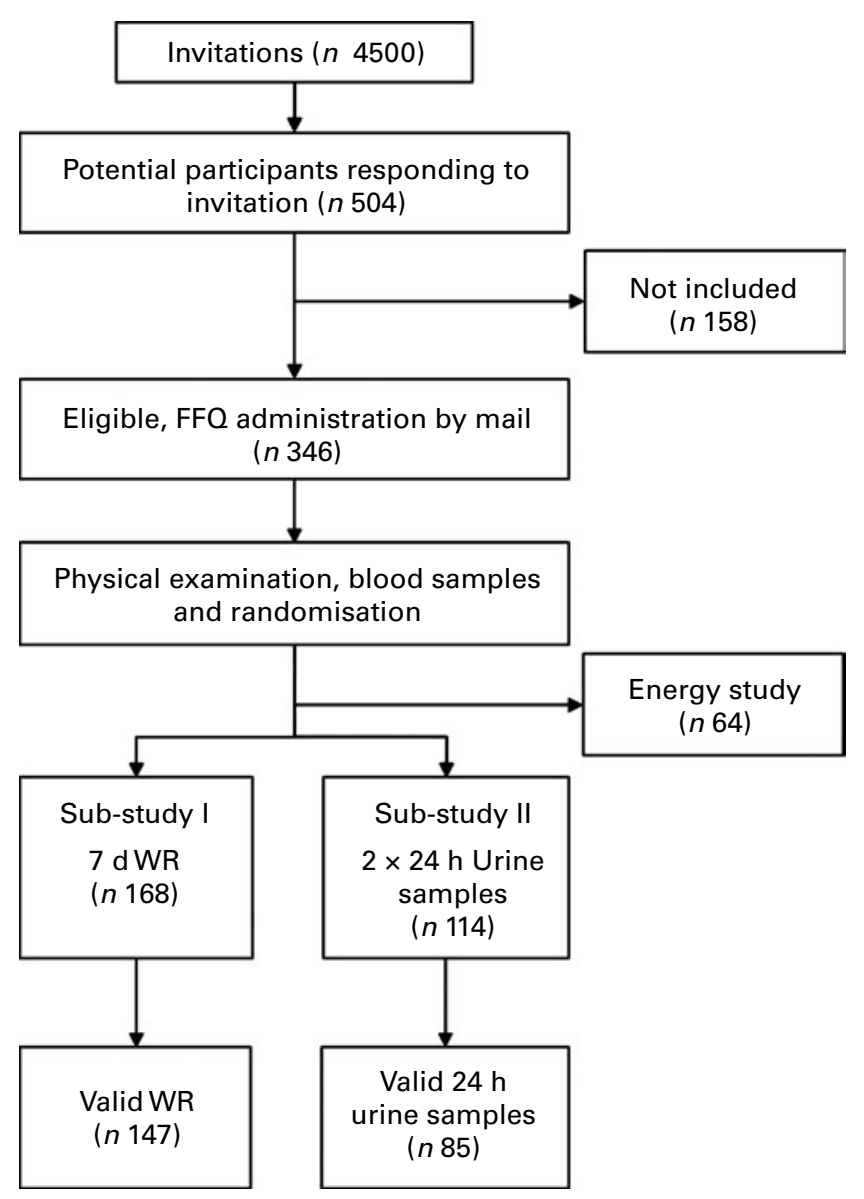

Fig. 1. Study design and flow chart. Of the 504 respondents, 346 were found to be eligible for participation. Randomisation into three sub-studies resulted in 168, 114 and sixty-four participants assigned to carry out weighed food records (WR), $24 \mathrm{~h}$ urine sampling or energy expenditure measurements, respectively. Only the results from sub-studies I and II are included in the present study.

nineteen questions about different berries, four about fruit, six about vegetables, two about chocolate, ten about nuts and seeds, twenty-seven about spices and herbs, three about coffee and two about tea intake. Questions concerning seasonal variation in berry intake were also included. The options of frequency of consumption of particular food items ranged from several times a day to once a month, with options of portion sizes based on household units such as slices, glasses, cups, pieces, spoons and teaspoons. The amounts of the portion sizes were based on portions estimated by WR from previous surveys ${ }^{(31)}$. The FFQ was scanned, and the image files were transferred into data files using Cardiff Teleform 2006 software. The data files were checked for completeness, and daily intakes of fruit and vegetables were computed using the food database AE-07 and KBS software system (KBS, version 4.9, 2008) developed at the Department of Nutrition, University of Oslo, Norway. The food database AE-07 is based on the 2006 edition of the Norwegian food composition table (www.norwegianfoodcomp.no). In the present study, fruit intake is the sum of fresh, dried and canned fruit and fruit juice intake. Berries and berry products were not 
included. The intake of vegetables was assessed by twentyseven questions about different vegetables, including vegetables in compound dishes such as casseroles, pizza, tacos and soups. Vegetables from compound dishes were estimated from standardised recipes.

\section{The $7 d$ weighed food records}

The WR registration was divided into two periods of four and three subsequent days, $7 \mathrm{~d}$ apart, covering all days of a week. The participants were equipped with a food registration diary and a scale (Phillips 2393/95, 1-5 kg) and were instructed to weigh and register everything that they ate and drank during the registration period. After completion, the records were returned to the Department of Nutrition, University of Oslo, by mail. Daily intakes of fruit and vegetables were computed using the food database AE-07 and KBS software system (KBS, version 4.9, 2008) and included all vegetables reported as single items or as part of mixed dishes. The records were checked for completeness, and twenty-one participants were excluded due to incomplete registrations. Thus, WR data from 147 participants were included in the analyses.

\section{Blood and urine samples}

Overnight fasting blood samples were collected, and plasma, serum and WBC samples were immediately prepared and stored at $-70^{\circ} \mathrm{C}$ unless immediately analysed. Urine samples were collected twice, 6 months apart; one sample was collected on a weekday and the other on a weekend. All weekdays were evenly represented; for the weekend days, more urine samples were sampled on Saturday than on Sunday. The participants were asked to discard the first morning urine and from then on to collect all urine for $24 \mathrm{~h}$ up to and including the morning urine the next day. The urine samples were collected in $3000 \mathrm{ml}$ dark plastic bottles containing $240 \mathrm{mg}$ ascorbic acid powder. After the first void of urine, the participants added $10 \mathrm{ml}$ of $1 \mathrm{M}-\mathrm{HCl}$ as the stabilising agent. The urine samples were brought to the laboratory by the staff. Total volume was registered, and $10 \mathrm{ml}$ aliquots of the samples were stored at $-70^{\circ} \mathrm{C}$ until analysis. The completeness of the urine samples was verified using the para-amino benzoic acid (PABA) method ${ }^{(37,38)}$. The participants took three tablets each containing $80 \mathrm{mg}$ PABA: one tablet at breakfast, one at the mid-day meal and one at the evening meal. PABA in the urine was measured using a colorimetric method ${ }^{(37)}$. Urine samples containing $80-110 \%$ of the PABA consumed were classified as acceptable ${ }^{(39)}$, whereas samples outside this range were not included in further analyses. Acceptable PABA checks in urine samples from both time points were found for eighty-five participants.

\section{Determination of flavonoids in urine}

The flavonoids apigenin, eriodictyol, hesperidin, kaempferol, naringenin, phloretin, quercetin and tamarixetin/isorhamnetin were quantified in the urine samples by HPLC-atmospheric pressure chemical ionisation-MS/MS at AS Vitas (Oslo, Norway; www.vitas.no). The procedure was adapted from Nielsen et $a l^{(40)}$ with minor modifications. In brief, $2 \mathrm{ml}$ urine were filtered, and internal standards were added and enzymatically treated with glucuronidase and sulphatase to remove sugars conjugated to the flavonols. After incubation $\left(45^{\circ} \mathrm{C}, 500 \mathrm{rpm}\right.$ for $1 \mathrm{~h}$ ) and subsequent cooling to room temperature, residual proteins were precipitated by the addition of ice-cold methanol and centrifugation. After evaporation with hot $\mathrm{N}_{2}$, the residue was dissolved in $150 \mu \mathrm{l}$ of $35 \%$ methanol in water. Then, $100 \mu \mathrm{l}$ was injected directly into the HPLC-MS/MS system. The mean value for both urine samples was used in the subsequent validation analyses.

\section{Determination of plasma carotenoids}

$\alpha$-Carotene, $\beta$-carotene, $\beta$-cryptoxanthin, lutein, lycopene and zeaxanthin in plasma were determined by HPLC. Proteins were precipitated and removed by the addition of 4.5 volumes of isopropanol followed by centrifugation at $3000 \mathbf{g}$ at $4^{\circ} \mathrm{C}$ for $15 \mathrm{~min}$. The internal standard astaxanthin was added with isopropanol. The clear supernatant $(25 \mu \mathrm{l})$ was used for analysis. The mobile phases consisted of A (20\% water and $24 \%$ acetone in ethanol) and B (acetone). The gradient conditions were as follows: from 2 to $100 \%$ B within $20 \mathrm{~min}$, followed by $100 \%$ B for $15 \mathrm{~min}$. Detection was performed at $453 \mathrm{~nm}$ using a variable wavelength detector. Plasma calibrators quantified against the National Institute of Standards and Technology 968c Standardized Reference material served as standards.

\section{Statistical methods}

Age and BMI were normally distributed and are presented as means. Plasma carotenoid concentrations, amount of urinary flavonoids and fruit and vegetable intakes were not normally distributed and are presented as medians. Differences in intake estimates from the FFQ and the WR were tested using non-parametric statistical methods. To investigate the relationship between the intakes from the FFQ and the WR, the Spearman correlation coefficient was calculated. To investigate the relationship between fruit and vegetable intakes and biomarkers, data were ln-transformed, and Pearson's correlation coefficients were calculated for intakes from the FFQ and urinary flavonoids and for the intakes estimated from the FFQ and the WR. The correlations between plasma carotenoid concentrations and the other variables were performed using partial correlation, adjusting for BMI. Correlations are presented with $95 \% \mathrm{CI}^{(41)}$.

The method of triads was used to estimate the validity coefficients (VC) between theoretical true intake and intakes estimated from the FFQ, the WR and the biomarkers ${ }^{(15,29)}$. Let $\mathrm{Q}, \mathrm{R}$ and $\mathrm{M}$ be the measurements of dietary intake from the FFQ, the WR and the biomarker, respectively, and let T denote the true unknown long-term dietary intake. When using two biomarkers, let $\mathrm{R}$ be the first and $\mathrm{M}$ be the second 
biomarker. The VC can be estimated as follows:

$$
\begin{aligned}
& \mathrm{VC}_{\mathrm{QT}}=\sqrt{\left(\left(r_{\mathrm{QR}} \times r_{\mathrm{QM}}\right) / r_{\mathrm{RM}}\right),} \\
& \mathrm{VC}_{\mathrm{RT}}=\sqrt{\left(\left(r_{\mathrm{QR}} \times r_{\mathrm{RM}}\right) / r_{\mathrm{QM}}\right),} \\
& \mathrm{VC}_{\mathrm{MT}}=\sqrt{\left(\left(r_{\mathrm{QM}} \times r_{\mathrm{RM}}\right) / r_{\mathrm{QR}}\right)},
\end{aligned}
$$

where $r$ is the correlation coefficient ${ }^{(15,29)}$. This technique assumes that random errors in the dietary assessments are uncorrelated, and that positive linear correlation exists between estimates of the dietary intake and true intake ${ }^{(29,42)}$. The 95\% CI for the estimates of the VC were obtained using the percentile bootstrap method ${ }^{(29,43)}$ with 1000 bootstrap samples. The $95 \%$ CI are thus based on the intervals from the 2.5 to the 97.5 percentile of the $\mathrm{VC}$ found by using the 1000 bootstrap samples. A well-known problem with the method of triads is that the VC estimates may become larger than $1^{(15)}$. Another problem is that the VC cannot be estimated using equations (1)-(3) due to the square root in the formulas, when the estimated correlation is negative. None of these problems were present when estimating the VC from our data, but they were for several of the bootstrap samples. The reason for these problems is the fairly crude estimation method. To avoid these problems, we used maximumlikelihood estimation for factor analysis models, which is the basis for the method of triads ${ }^{(44)}$. Thus, when calculating the $\mathrm{CI}$, the VC for the bootstrap samples were found based on maximum-likelihood estimation. Both the maximumlikelihood estimation and the bootstrap analyses were performed using open-source statistical software $\mathrm{R}$, version 2.7.0 ${ }^{(45)}$. All other statistical analyses were performed using SPSS for Windows release 16.0 (SPSS, Inc., Chicago, IL, USA). Results were considered to be statistically significant at $P<0 \cdot 05$. Significant levels are two-sided.

Based on results from other recent validation studies using plasma carotenoids and urinary flavonoids as biomarkers of fruit and vegetable intake ${ }^{(18-22,25,26)}$, as well as the observed correlations in the present sub-studies, the following combinations of biomarkers were used in the method of triads: $\beta$-cryptoxanthin as the biomarker for fruit (including juice) intake; the sum of $\alpha$-carotene and $\beta$-carotene as the biomarker for vegetable intake; the sum of $\alpha$-carotene, $\beta$-carotene and $\beta$-cryptoxanthin as well as the sum of urinary flavonoids as the biomarkers of fruit, juice and vegetable intake. Because of the lack of a valid biomarker for the intake of berries and berry products, these intakes were excluded from the calculations.

\section{Results}

A total of 282 subjects were included in sub-studies I and II (Fig. 1). After completion of data sampling, we had valid datasets from 147 participants in sub-study I and from 85 in sub-study II, of whom 110 were male and thirty-two were current smokers. Mean age and BMI were 47 (95\% CI 45, 49) years and $24 \cdot 7(95 \% \mathrm{CI} 24 \cdot 2,25 \cdot 2) \mathrm{kg} / \mathrm{m}^{2}$, respectively. Intakes of fruit (including juice) and vegetables from the FFQ and the WR and plasma carotenoid concentrations from sub-study I are presented in Table 1 . The total intake of fruit (including juice) did not differ significantly between the FFQ and the WR $(P=0.74)$. In contrast, intake of vegetables was significantly higher when estimated by the FFQ compared with the WR $(P<0 \cdot 001)$; the median absolute difference between the two methods was $67 \mathrm{~g} / \mathrm{d}$ or $39 \%$ of WR intake. Furthermore, total intake of fruit and vegetables was significantly higher when estimated by the FFQ than by the WR $(P<0 \cdot 001)$. Spearman's correlations between the two dietary measurement methods were $0.57(P=0.000), 0.38(P=0.000)$ and $0.49(P=0.000)$ for intake of fruit (including juice), vegetables and total fruit, juice and vegetable intake, respectively.

Correlations between plasma carotenoids and intakes from the FFQ and the WR are given in Table 2. For most of the correlations, the intakes of fruit and vegetables from the FFQ showed higher correlations with the plasma carotenoids than the intakes from the WR. Significant correlations were observed between intakes of fruit (including juice) from both the FFQ and the WR and plasma $\beta$-cryptoxanthin, whereas intakes of vegetables from the FFQ and the WR correlated significantly with plasma $\alpha$-carotene and $\beta$-carotene concentrations. The total intake of fruit, juice and vegetables from the FFQ showed stronger correlations and correlated with more plasma carotenoids than the corresponding intake estimates from the WR (Table 2).

Intakes of fruit (including juice) and vegetables from the FFQ, plasma carotenoid concentrations, urinary flavonoids and corresponding correlations in sub-study II are presented in Table 3. The intake of fruit (including juice) showed significant correlations with apigenin, eriodictyol, hesperidin, naringenin, tamarixetin/isorhamnetin and total flavonoids in urine. Vegetable intake correlated significantly with apigenin, tamarixetin/isorhamnetin and total flavonoids in urine, whereas total intake of fruit, juice and vegetables correlated significantly with most of the urinary flavonoids (Table 3).

Table 1. Intakes of fruit and vegetables estimated from the FFQ and the $7 \mathrm{~d}$ weighed food records (WR) and plasma carotenoid concen-

\begin{tabular}{|c|c|c|}
\hline & Median & Interquartile range \\
\hline \multicolumn{3}{|l|}{ FFQ intakes $(g / d)$} \\
\hline Fruit and juice & 220 & $147-368$ \\
\hline Vegetables & 243 & $166-332$ \\
\hline Fruit, juice and vegetables & 513 & $354-644$ \\
\hline \multicolumn{3}{|l|}{ WR intakes $(g / d)$} \\
\hline Fruit and juice & 248 & $117-363$ \\
\hline Vegetables & 161 & $118-222$ \\
\hline Fruit, juice and vegetables & 418 & $269-591$ \\
\hline \multicolumn{3}{|l|}{ Plasma carotenoids (nmol/l) } \\
\hline$\alpha$-Carotene & 98 & $57-168$ \\
\hline$\beta$-Carotene & 414 & $274-616$ \\
\hline$\beta$-Cryptoxanthin & 141 & $83-211$ \\
\hline Lutein & 156 & $122-209$ \\
\hline Lycopene & 567 & $403-758$ \\
\hline Zeaxanthin & 39 & $28-53$ \\
\hline Total carotenoids & 1529 & $1156-2031$ \\
\hline
\end{tabular}
trations in sub-study I

(Median values and interquartile ranges, $n$ 147) 
All plasma carotenoids except lycopene showed significant correlations with intakes of fruit (including juice) and total fruit, juice and vegetable intake (Table 3). However, intake of vegetables correlated significantly only with $\alpha$-carotene, $\beta$-carotene, lutein and total carotenoids.

The VC for the FFQ and the reference methods in both substudies are shown in Table 4. The VC for the intake of vegetables from the FFQ were 0.61 and 0.60 in sub-studies I and II, respectively. The corresponding VC for intake of fruit (including juice) from the FFQ were 0.75 and 0.67 , whereas the VC were 0.88 and 0.94 , respectively, for total intake of fruit, juice and vegetables from the FFQ.

Of the participants, thirty-four reported habitual use of food supplements containing different amounts of carotenoids and/ or flavonoids. No significant differences in plasma carotenoid concentrations or urinary flavonoids were observed between users and non-users of supplements, except for $\alpha$-carotene, which was significantly higher for users than for non-users $(P=0.04)$. Correlations between intake estimates and biomarkers did not change significantly with or without supplement users, and therefore supplement users were included in all analyses.

\section{Discussion}

The major strength of the present validation study is that we have applied two independent sets of biomarkers for the dietary intake of fruit and vegetables in combination with the method of triads. The average intake of fruit and juice from the FFQ was similar, whereas the average intake of vegetables was higher compared with the WR. The VC for intakes of fruit, juice and vegetables from the FFQ were high.

\section{Comparisons of FFQ and weighed food record estimates}

The FFQ provided similar data on median intake of fruit, whereas the intake of vegetables was higher compared with the WR. There may be several explanations for this. The questions concerning fruit and fruit juice consumption in the FFQ were presented in portion sizes such as pieces or glasses, which may be easier to recall than vegetable portion sizes, which may vary more, and often are integrated parts of whole dishes. In addition, the relatively large number of questions about different types of vegetables, nineteen in total, may lead to the observed high intake of vegetables. WR have been widely accepted as a preferred reference method in the validation of FFQ because the assessment errors are mostly independent ${ }^{(14,46)}$. However, prolonged recording may reduce the recorded intake because the registration itself may be too demanding for the subjects ${ }^{(47)}$, causing changes in behaviour and dietary intake ${ }^{(46)}$, and consequently under-reporting of food intake ${ }^{(46,47)}$. We did not observe any reduction in the intake of vegetables registered from day 1 to 7 in the WR; thus it seems unlikely that there was underreporting of vegetable intake in the WR due to participant burden. 
Table 3. Dietary intakes from the FFQ, flavonoids in $24 \mathrm{~h}$ urine samples and plasma carotenoid concentrations in sub-study II (Median values, interquartile ranges (IQR), correlation coefficients and $95 \%$ confidence intervals, $n$ 85)

\begin{tabular}{|c|c|c|c|c|c|c|c|c|}
\hline & \multirow[b]{2}{*}{ Median } & \multirow[b]{2}{*}{ IQR } & \multicolumn{2}{|c|}{ FJ } & \multicolumn{2}{|c|}{ V } & \multicolumn{2}{|c|}{ FJV } \\
\hline & & & $r$ & $95 \% \mathrm{Cl}$ & $r$ & $95 \% \mathrm{Cl}$ & $r$ & $95 \% \mathrm{Cl}$ \\
\hline \multicolumn{9}{|l|}{ FFQ intakes $(g / d)$} \\
\hline FJ & 235 & $139-362$ & & & & & & \\
\hline $\mathrm{V}$ & 222 & $157-370$ & & & & & & \\
\hline FJV & 489 & $357-705$ & & & & & & \\
\hline \multicolumn{9}{|c|}{ Excreted urinary flavonoids $\dagger(\mu \mathrm{g})$} \\
\hline Apigenin & 0.9 & $0.1-3.5$ & $0.23^{*}$ & $0.02,0.42$ & $0.26^{\star}$ & $0.05,0.45$ & $0.30^{\star \star}$ & $0.09,0.48$ \\
\hline Eriodictyol & 31.7 & $11 \cdot 6-62 \cdot 0$ & $0.27^{\star}$ & $0.06,0.46$ & 0.05 & $-0.16,0.26$ & $0.24^{*}$ & $0.03,0.43$ \\
\hline Hesperidin & 98.0 & $11 \cdot 4-516 \cdot 1$ & $0.53^{\star \star \star}$ & $0.36,0.67$ & 0.15 & $-0.07,0.35$ & $0.44^{\star \star *}$ & $0.25,0.60$ \\
\hline Kaempferol & 7.9 & $4 \cdot 1-15 \cdot 7$ & 0.19 & $-0.02,0.39$ & 0.07 & $-0.15,0.28$ & 0.19 & $-0.02,0.39$ \\
\hline Naringenin & 221.4 & $97 \cdot 3-560 \cdot 0$ & $0.49^{\star \star \star}$ & $0.31,0.64$ & 0.12 & $-0.10,0.32$ & $0.40^{\star \star \star}$ & $0.20,0.56$ \\
\hline Phloretin & $62 \cdot 7$ & $29 \cdot 8-117 \cdot 7$ & 0.11 & $-0.11,0.32$ & 0.01 & $-0.20,0.22$ & 0.11 & $-0.11,0.32$ \\
\hline Quercetin & $61 \cdot 2$ & $22 \cdot 8-155 \cdot 3$ & 0.03 & $-0.18,0.24$ & -0.02 & $-0.23,0.19$ & 0.01 & $-0.20,0.22$ \\
\hline Tamarixetin/isorhamnetin & 20.5 & $9 \cdot 5-44 \cdot 7$ & $0.46^{\star \star \star}$ & $0.27,0.61$ & $0 \cdot 26^{*}$ & $0.05,0.45$ & $0.47^{\star \star \star}$ & $0.29,0.62$ \\
\hline Total flavonoids & 650.5 & $323 \cdot 1-1457 \cdot 0$ & $0.45^{\star \star *}$ & $0.26,0.61$ & 0.11 & $-0.11,0.32$ & $0.39^{\star \star \star}$ & $0.19,0.56$ \\
\hline \multicolumn{9}{|l|}{ Plasma carotenoids $\ddagger$ (nmol/l) } \\
\hline$\alpha$-Carotene & $112 \cdot 0$ & $72 \cdot 0-174 \cdot 0$ & $0.45^{\star \star *}$ & $0.26,0.61$ & $0.37^{\star \star \star}$ & $0.17,0.54$ & $0.53^{\star \star \star}$ & $0.36,0.67$ \\
\hline$\beta$-Carotene & $501 \cdot 0$ & $307 \cdot 0-684 \cdot 0$ & $0.31^{* \star *}$ & $0.10,0.49$ & $0.29^{\star \star}$ & $0.08,0.47$ & $0.37^{\star \star \star}$ & $0.17,0.54$ \\
\hline$\beta$-Cryptoxanthin & $140 \cdot 0$ & $92 \cdot 0-22 \cdot 0$ & $0.49^{\star \star \star}$ & $0.31,0.64$ & 0.14 & $-0.07,0.34$ & $0.41^{\star \star \star}$ & $0.22,0.57$ \\
\hline Lutein & $163 \cdot 0$ & $117 \cdot 0-201 \cdot 0$ & $0.31^{\star \star}$ & $0.10,0.49$ & $0.24^{\star}$ & $0.03,0.43$ & $0.31^{\star \star}$ & $0.10,0.49$ \\
\hline Lycopene & $623 \cdot 0$ & $454 \cdot 0-780 \cdot 0$ & 0.12 & $-0.10,0.32$ & 0.17 & $-0.04,0.37$ & $0 \cdot 16$ & $-0.06,0.36$ \\
\hline Zeaxanthin & 39.0 & $29 \cdot 0-51 \cdot 0$ & $0.33^{\star}$ & $0.13,0.51$ & 0.05 & $-0.16,0.26$ & $0.25^{\star}$ & $0.04,0.44$ \\
\hline Total carotenoids & $1612 \cdot 0$ & $1287 \cdot 0-2008 \cdot 0$ & $0.43^{\star * *}$ & $0.24,0.59$ & $0 \cdot 34^{\star *}$ & $0.14,0.52$ & $0.46^{\star \star *}$ & $0.27,0.61$ \\
\hline
\end{tabular}

FJ, fruit and juice; V, vegetables; FJV, fruit, juice and vegetables.

Correlation coefficients were statistically significant: ${ }^{\star} P<0.05$, ${ }^{\star \star} P<0.01,{ }^{\star \star \star} P<0.001$

† Flavonoid correlations: In-transformed data, Pearson's correlations.

$\ddagger$ Carotenoid correlations: In-transformed data, partial correlation adjusted for BMI.

\section{Fruit and vegetable intake and plasma carotenoids}

The strongest correlations between intakes from the two dietary assessment methods and plasma carotenoids were observed between intakes of fruit and the plasma concentration of $\beta$-cryptoxanthin. Citrus fruit and juices are major dietary sources of $\beta$-cryptoxanthin ${ }^{(27)}$ and are frequently consumed by the participants in the present study population $(35 \%$ of the total fruit and vegetable consumption, data not shown). The positive association between plasma $\beta$-cryptoxanthin and the intake of fruit and vegetables in the present study is in agreement with earlier studies, showing $\beta$-cryptoxanthin to be a consistent biomarker for fruit and vegetable intake ${ }^{(19,21,23,47,48)}$. Intake of vegetables from the FFQ showed good and significant correlations with $\alpha$-carotene and $\beta$-carotene, in accordance with earlier studies ${ }^{(17,23,49,50)}$. An earlier Norwegian study showed a proportionally larger response in serum concentrations of $\alpha$-carotene than other carotenoids to variations in fruit and vegetable consumption ${ }^{(19)}$. Important sources of $\alpha$-carotene are carrots, red and green pepper and bananas ${ }^{(27)}$; all are frequently consumed in Norway.

\section{Fruit and vegetable intake and flavonoids in urine}

Earlier dietary intervention studies have shown that small changes in the intake of fruit and vegetable are reflected in the amount of flavonoids excreted in urine ${ }^{(18,20,22,25,26)}$. In the present study, the amount of total urinary flavonoids showed good correlation with the total intake of fruit, juice and vegetables. The highest correlations were observed between the

Table 4. Validity coefficients (VC) for the FFQ, weighed food records (WR), plasma carotenoid concentrations and urinary flavonoids in sub-studies I and II

(Validity coefficients and $95 \%$ confidence intervals)

\begin{tabular}{|c|c|c|c|c|c|c|}
\hline & \multicolumn{2}{|c|}{ FJ } & \multicolumn{2}{|r|}{ V } & \multicolumn{2}{|c|}{ FJV } \\
\hline & VC & $95 \% \mathrm{Cl}$ & VC & $95 \% \mathrm{Cl}$ & VC & $95 \% \mathrm{Cl}$ \\
\hline \multicolumn{7}{|l|}{ Sub-study I ( $n$ 147) } \\
\hline FFQ (T) & 0.75 & $0.56,0.93$ & 0.61 & $0.35,0.98$ & 0.88 & $0.58,0.98$ \\
\hline WR (T) & 0.77 & $0.59,0.99$ & 0.51 & $0.29,0.72$ & 0.50 & $0.37,0.71$ \\
\hline Plasma carotenoids $(\mathrm{T})$ & 0.49 & $0.31,0.66$ & 0.54 & $0.36,0.72$ & 0.38 & $0.22,0.59$ \\
\hline \multicolumn{7}{|l|}{ Sub-study II ( $n$ 85) } \\
\hline FFQ $(T)$ & 0.67 & $0.51,0.80$ & 0.60 & $0.19,0.98$ & 0.94 & $0.64,0.98$ \\
\hline Urinary flavonoids $(T)$ & 0.67 & $0.48,0.84$ & $0 \cdot 17$ & $-0.18,0.49$ & 0.41 & $0.23,0.63$ \\
\hline Plasma carotenoids $(T)$ & 0.70 & $0.60,0.92$ & 0.53 & $0.17,0.98$ & 0.47 & $0.28,0.72$ \\
\hline
\end{tabular}

FJ, fruit and juice; V, vegetables; FJV, fruit, juice and vegetables; T, true intake. 
intake of fruit (including juice) and the amount of the two citrus flavonoids hesperidin and naringenin in urine, which are specific biomarkers for grapefruit and orange intake ${ }^{(24,51,52)}$. Furthermore, the observed values of urinary flavonoids in the present study were in the same range as reported from earlier intervention studies $^{(18,20,22)}$, thus showing that the FFQ can discriminate between intakes in the normal everyday range of fruit and vegetable consumption.

\section{The method of triads and validity coefficients}

Originally, the method of triads was used with one biomarker and two dietary assessment methods ${ }^{(15,17,29,42,53)}$. However, the assumption of total independence between the errors of the two dietary assessment methods was in many cases unrealistic $^{(54)}$. The present study is one of a few studies ${ }^{(44)}$ combining two independent and specific biomarkers of fruit and vegetable intake when validating the intakes of fruit and vegetables from an FFQ and applying the method of triads. The main advantage of dietary biomarkers is that they are objective measures and independent of the biases and errors associated with dietary assessment methods ${ }^{(15,16,29)}$. In the present study, the triad testing showed that the VC were high for the intakes from the FFQ. We also observed generally higher VC for the FFQ than for the WR and the plasma and urinary biomarkers. The $\mathrm{VC}$ for the plasma carotenoids were, with one exception, lower than the $\mathrm{VC}$ for the dietary assessment methods. Andersen et al. ${ }^{(17)}$ also observed this when using an FFQ and WR together with plasma carotenoids; the VC for the assessment methods often were higher than the VC for the biomarker. This may be explained by individual differences in digestion, absorption, distribution, metabolism and excretions of carotenoids ${ }^{(16)}$. We speculate that the lower VC for the intake of vegetables from the FFQ compared with the intake of fruit and juice observed in the present sub-studies may reflect difficulties associated with reporting vegetable portion sizes and frequency of consumption, especially for vegetables that are part of mixed dishes.

\section{Strengths and limitations of the validity study}

The strength of the present validation study is the use of two biomarkers as independent reference methods for the assessment of the VC. Moreover, we used the mean of two $24 \mathrm{~h}$ urine samples for the determination of urinary flavonoids, instead of a single urine sample. By using independent reference methods and repeated measures of biomarkers, we tried to optimise the measures of the reference methods for application in the method of triads.

The biomarkers used in the present study are concentration biomarkers; thus they cannot be translated into absolute levels of intake ${ }^{(55)}$ but are instead used to evaluate the relative intakes of fruit, juice and vegetables. However, when using biomarkers of nutrient intake, it is important to keep in mind all the factors that influence the concentration of the biomarker. The intake of specific flavonoids and carotenoids varies widely between countries and cultures ${ }^{(56,57)}$. Thus, different sets of biomarkers may reflect different dietary sources in different studies, complicating direct comparison of results and designs across studies and countries. The bioavailability of carotenoids is influenced by systemic factors such as age, sex, genotype, physiological state, chronic or acute disease and intestinal digestion and absorption ${ }^{(28,50,58,59)}$ in addition to food processing and diet composition ${ }^{(27,60)}$. Plasma carotenoid concentrations are also influenced by smoking ${ }^{(61)}$, as well as by intake of carotenoid supplements ${ }^{(27)}$. Likewise, the bioavailability of flavonoids varies with food matrix, genetic profile and the composition of intestinal microflora ${ }^{(62)}$. Moreover, the absorption of flavonoids varies between individual flavonoids, depending on the molecular structure ${ }^{(56)}$, and the citrus flavanoids are more accessible to absorption and excretion than many other flavonoids ${ }^{(56)}$. The good association observed for hesperidin and naringenin in contrast to the poor association observed for quercetin in the present study is in agreement with previous studies ${ }^{(56)}$.

The invited study population was a random sample of the Norwegian adult population, but the participation rate was low. The age distribution in the study population was not significantly different from the general population but included fewer smokers (3\%) and slightly more women than in the general population (2\%). The last nationwide dietary survey in Norway in $1997^{(32)}$ showed that the average daily intake of fruit, berries, juice and vegetables was $357 \mathrm{~g} / \mathrm{d}$, whereas the corresponding numbers were approximately 500 and $420 \mathrm{~g} / \mathrm{d}$, estimated from the FFQ and the WR, respectively. This discrepancy is probably due to a higher intake of fruit and vegetables among our participants than the general population.

It is important to note the different reference periods for test and reference methods. The FFQ considers intake during the preceding year, the WR during $7 \mathrm{~d}$, the plasma carotenoids mostly represent the preceding weeks of dietary exposure ${ }^{(58)}$, whereas the urinary flavonoids represent an even shorter time period, of days and hours ${ }^{(63)}$. The observed half lives of plasma carotenoids suggest that repeated consumption of carotenoids results in fairly constant plasma carotenoid patterns for up to 1 year ${ }^{(64)}$. MacDonald et al $^{(65)}$ observed that $\beta$-carotene and $\beta$-cryptoxanthin were suitable markers of long-term fruit and vegetable intake for up to 2 years. Despite different time frames, significant correlations were observed between the FFQ and the two biomarkers in the present study. However, higher correlations might have been found with an increased number of urine and blood samples, distributed over the same time period as covered by the FFQ. Overall, the present results suggest that the use of two independent biomarkers of fruit and vegetable intake in the method of triads is suitable for the evaluation of dietary assessments.

\section{Conclusions}

The FFQ provided estimates of fruit intake similar to the intakes from the WR. Moreover, based on the positive associations between the FFQ estimates, the biomarkers and the WR and the FFQ VC, the FFQ was found valid and suitable for ranking of subjects according to their usual intake of fruit and vegetables. Thus, the FFQ may be implemented as a dietary assessment tool for measuring fruit, juice and vegetable intake in future nutritional epidemiological studies. 


\section{Acknowledgements}

The present study was supported by Aktieselskabet Freia Chocolate Fabrik's Medical Foundation, Throne-Holst Foundation for Nutrition Research, the Research Council of Norway and the Norwegian Cancer Society. Elin Bjørge Løken and Jannicke Fredriksen are acknowledged for assistance with the Food Database AE-07; Gunnar Amlid for valuable help with Cardiff Teleform 2006 software; Vitas AS and Thomas Gundersen for the analyses of flavonoids in urine. R. B. and C. A. D. are shareholders in Vitas AS. The other authors declare that they have no competing interests. M. H. C. carried out the redesign of the FFQ, study design, data collection, statistical analyses and preparation of the manuscript. I. T. L. L. contributed to the study design, data collection and manuscript revision. A. K. carried out the analyses of plasma carotenoids and contributed to the study design, data collection and manuscript revision. J. M. G. estimated the CI in the method of triads. R. B. and C. A. D. contributed to funding, study design and manuscript revision. L. F. A. contributed to the study design, design of the FFQ, statistical analyses and manuscript revision. All authors read and approved the final manuscript.

\section{References}

1. Andersen LF, Jacobs DR Jr, Carlsen MH, et al. (2006) Consumption of coffee is associated with reduced risk of death attributed to inflammatory and cardiovascular diseases in the Iowa Women's Health Study. Am J Clin Nutr 83, 1039-1046.

2. Blomhoff R, Carlsen MH, Andersen LF, et al. (2006) Health benefits of nuts: potential role of antioxidants. Br J Nutr 96, Suppl. 2, S52-S60.

3. Jacobs DR Jr, Andersen LF \& Blomhoff R (2007) Whole-grain consumption is associated with a reduced risk of noncardiovascular, noncancer death attributed to inflammatory diseases in the Iowa Women's Health Study. Am J Clin Nutr 85, 1606-1614.

4. Joshipura KJ, Hu FB, Manson JE, et al. (2001) The effect of fruit and vegetable intake on risk for coronary heart disease. Ann Intern Med 134, 1106-1114.

5. Joshipura KJ, Ascherio A, Manson JE, et al. (1999) Fruit and vegetable intake in relation to risk of ischemic stroke. JAMA 282, 1233-1239.

6. Karlsen A, Retterstol L, Laake P, et al. (2007) Anthocyanins inhibit nuclear factor-kappaB activation in monocytes and reduce plasma concentrations of pro-inflammatory mediators in healthy adults. J Nutr 137, 1951-1954.

7. Malik A, Afaq F, Sarfaraz S, et al. (2005) Pomegranate fruit juice for chemoprevention and chemotherapy of prostate cancer. Proc Natl Acad Sci U S A 102, 14813-14818.

8. World Cancer Research Fund \& American Institute for Cancer Research (2007) Food, Nutrition, Physical Activity and the Prevention of Cancer: A Global Perspective. Washington, DC: AICR.

9. Wright ME, Mayne ST, Swanson CA, et al. (2003) Dietary carotenoids, vegetables, and lung cancer risk in women: the Missouri women's health study (United States). Cancer Causes Control 14, 85-96.

10. Cabrera C, Artacho R \& Gimenez R (2006) Beneficial effects of green tea - a review. J Am Coll Nutr 25, 79-99.

11. Cooper KA, Donovan JL, Waterhouse AL, et al. (2008) Cocoa and health: a decade of research. Br J Nutr 99, 1-11.
12. deKok TM, van Breda SG \& Manson MM (2008) Mechanisms of combined action of different chemopreventive dietary compounds. Eur J Nutr 47, 51-59.

13. Wang L-S \& Stoner GD (2008) Anthocyanins and their role in cancer prevention. Cancer Lett 269, 281-290.

14. Willett W (1998) Nutritional Epidemiology, 2nd ed. Oxford: Oxford University Press.

15. Ocke MC \& Kaaks RJ (1997) Biochemical markers as additional measurements in dietary validity studies: application of the method of triads with examples from the European Prospective Investigation into Cancer and Nutrition. Am J Clin Nutr 65, 1240S-1245S.

16. Jenab M, Slimani N, Bictash M, et al. (2009) Biomarkers in nutritional epidemiology: applications, needs and new horizons. Hum Genet 125, 507-525.

17. Andersen LF, Veierod MB, Johansson L, et al. (2005) Evaluation of three dietary assessment methods and serum biomarkers as measures of fruit and vegetable intake, using the method of triads. BrJ Nutr 93, 519-527.

18. Brevik A, Rasmussen SE, Drevon CA, et al. (2004) Urinary excretion of flavonoids reflects even small changes in the dietary intake of fruits and vegetables. Cancer Epidemiol Biomarkers Prev 13, 843-849.

19. Brevik A, Andersen LF, Karlsen A, et al. (2004) Six carotenoids in plasma used to assess recommended intake of fruits and vegetables in a controlled feeding study. Eur J Clin Nutr 58, 1166-1173.

20. Krogholm KS, Haraldsdottir J, Knuthsen P, et al. (2004) Urinary total flavonoid excretion but not 4-pyridoxic acid or potassium can be used as a biomarker for the intake of fruits and vegetables. J Nutr 134, 445-451.

21. Jansen MC, Van Kappel AL, Ocke MC, et al. (2004) Plasma carotenoid levels in Dutch men and women, and the relation with vegetable and fruit consumption. Eur J Clin Nutr $\mathbf{5 8}$, $1386-1395$.

22. Nielsen SE, Freese R, Kleemola P, et al. (2002) Flavonoids in human urine as biomarkers for intake of fruits and vegetables. Cancer Epidemiol Biomarkers Prev 11, 459-466.

23. Campbell DR, Gross MD, Martini MC, et al. (1994) Plasma carotenoids as biomarkers of vegetable and fruit intake. Cancer Epidemiol Biomarkers Prev 3, 493-500.

24. Ito H, Gonthier MP, Manach C, et al. (2005) Polyphenol levels in human urine after intake of six different polyphenol-rich beverages. Br J Nutr 94, 500-509.

25. Mennen LI, Sapinho D, Ito H, et al. (2006) Urinary flavonoids and phenolic acids as biomarkers of intake for polyphenolrich foods. Br J Nutr 96, 191-198.

26. Mennen LI, Sapinho D, Ito H, et al. (2008) Urinary excretion of 13 dietary flavonoids and phenolic acids in free-living healthy subjects - variability and possible use as biomarkers of polyphenol intake. Eur J Clin Nutr 62, 519-525.

27. Maiani G, Caston MJP, Catasta G, et al. (2009) Carotenoids: actual knowledge on food sources, intakes, stability and bioavailability and their protective role in humans. Mol Nutr Food Res 53, s194-s218.

28. Al-Delaimy WK, Slimani N, Ferrari P, et al. (2005) Plasma carotenoids as biomarkers of intake of fruits and vegetables: ecological-level correlations in the European Prospective Investigation into Cancer and Nutrition (EPIC). Eur J Clin Nutr 59, 1397-1408.

29. Kaaks RJ (1997) Biochemical markers as additional measurements in studies of the accuracy of dietary questionnaire measurements: conceptual issues. Am J Clin Nutr $\mathbf{6 5}$, 1232S-1239S.

30. Carlsen MH, Lillegaard IT, Karlsen A, et al. (2010) Evaluation of energy and dietary intake estimates from a food frequency 
questionnaire using independent expenditure measurement and weighed food records. Nutr J 9, 37.

31. Solvoll K (2000) Development, evaluation and application of a quantitative food frequency questionnaire for assessment of dietary habits. Thesis, University of Oslo.

32. Johansson L \& Solvoll K (1999) Norkost 1997, Landsomfattende kostholdundersøkelse blant menn og kvinner i alderen 16-79 år. no. 2 (Nationwide Dietary Survey Among Men and Women Aged 16- 79 Years). Oslo: Statens råd for ernæring sog fysisk aktivitet.

33. Nes M, Andersen LF, Solvoll K, et al. (1992) Accuracy of a quantitative food frequency questionnaire applied in elderly Norwegian women. Eur J Clin Nutr 46, 809-821.

34. Andersen LF, Tomten H, Haggarty P, et al. (2003) Validation of energy intake estimated from a food frequency questionnaire: a doubly labelled water study. Eur J Clin Nutr 57, 279-284

35. Andersen LF, Solvoll K, Johansson LR, et al. (1999) Evaluation of a food frequency questionnaire with weighed records, fatty acids, and alpha-tocopherol in adipose tissue and serum. Am J Epidemiol 150, 75-87.

36. Carlsen MH, Halvorsen BL, Holte K, et al. (2010) The total antioxidant content of more than 3100 foods, beverages, spices, herbs and supplements used worldwide. Nutr J 9, 3 .

37. Bingham S \& Cummings JH (1983) The use of 4-aminobenzoic acid as a marker to validate the completeness of $24 \mathrm{~h}$ urine collections in man. Clin Sci (Lond) 64, 629-635.

38. Bingham SA, Cassidy A, Cole TJ, et al. (1995) Validation of weighed records and other methods of dietary assessment using the $24 \mathrm{~h}$ urine nitrogen technique and other biological markers. Br J Nutr 73, 531-550.

39. Jakobsen J, Pedersen AN \& Ovesen L (2003) Para-aminobenzoic acid (PABA) used as a marker for completeness of 24 hour urine: effects of age and dosage scheduling. Eur $J$ Clin Nutr 57, 138-142.

40. Nielsen SE, Freese R, Cornett C, et al. (2000) Identification and quantification of flavonoids in human urine samples by column-switching liquid chromatography coupled to atmospheric pressure chemical ionization mass spectrometry. Anal Chem 72, 1503-1509.

41. Field A (2009) Discovering Statistics Using SPSS, 3rd ed. London: SAGE Publications Ltd.

42. Daures JP, Gerber M, Scali J, et al. (2000) Validation of a food-frequency questionnaire using multiple-day records and biochemical markers: application of the triads method. J Epidemiol Biostat 5, 109-115.

43. Efron B \& Tibshirani RJ (1993) An Introduction to the Bootstrap. Boca Raton, FL: Chapman \& Hall/CRC.

44. Brantsaeter AL, Haugen M, Rasmussen SE, et al. (2007) Urine flavonoids and plasma carotenoids in the validation of fruit, vegetable and tea intake during pregnancy in the Norwegian Mother and Child Cohort Study (MoBa). Public Health Nutr 10, 838-847.

45. Development Core Team (2008) $R$ : A Language and Environment for Statistical Computing. Vienna: R Foundation for Statistical Computing.

46. Cade J, Thompson R, Burley V, et al. (2002) Development, validation and utilisation of food-frequency questionnaires - a review. Public Health Nutr 5, 567-587.

47. Kristjansdottir AG, Andersen LF, Haraldsdottir J, et al. (2006) Validity of a questionnaire to assess fruit and vegetable intake in adults. Eur J Clin Nutr 60, 408-415.
48. Block G, Norkus E, Hudes M, et al. (2001) Which plasma antioxidants are most related to fruit and vegetable consumption? Am J Epidemiol 154, 1113-1118.

49. Van Kappel AL, Steghens JP, Zeleniuch-Jacquotte A, et al. (2001) Serum carotenoids as biomarkers of fruit and vegetable consumption in the New York Women's Health Study. Public Health Nutr 4, 829-835.

50. Al-Delaimy WK, Ferrari P, Slimani N, et al. (2005) Plasma carotenoids as biomarkers of intake of fruits and vegetables: individual-level correlations in the European Prospective Investigation into Cancer and Nutrition (EPIC). Eur J Clin Nutr 59, 1387-1396.

51. Gattuso G, Barreca D, Gargiulli C, et al. (2007) Flavonoid composition of citrus juices. Molecules 12, 1641-1673.

52. Erlund I, Meririnne E, Alfthan G, et al. (2001) Plasma kinetics and urinary excretion of the flavanones naringenin and hesperetin in humans after ingestion of orange juice and grapefruit juice. J Nutr 131, 235-241

53. McNaughton SA, Marks GC, Gaffney P, et al. (2005) Validation of a food-frequency questionnaire assessment of caroteinoid and vitamin $\mathrm{E}$ intake using weighed food records and plasma biomarkers: the method of triads model. Eur J Clin Nutr 59, 211-218.

54. Kaaks R \& Ferrari P (2006) Dietary intake assessments in epidemiology: can we know what we are measuring? Ann Epidemiol 16, 377-380.

55. Bingham S, Luben R, Welch A, et al. (2008) Associations between dietary methods and biomarkers, and between fruits and vegetables and risk of ischaemic heart disease, in the EPIC Norfolk Cohort Study. Int J Epidemiol 37, 978-987.

56. Rasmussen SE \& Breinholt VM (2003) Non-nutritive bioactive food constituents of plants: bioavailability of flavonoids. Int J Vitam Nutr Res 73, 101-111.

57. O'Neill ME, Carroll Y, Corridan B, et al. (2001) A European carotenoid database to assess carotenoid intakes and its use in a five-country comparative study. Br J Nutr $\mathbf{8 5}$, 499-507.

58. Castenmiller JJ \& West CE (1998) Bioavailability and bioconversion of carotenoids. Annu Rev Nutr 18, 19-38.

59. Gibson RS (2007) The role of diet- and host-related factors in nutrient bioavailability and thus in nutrient-based dietary requirement estimates. Food Nutr Bull 28, S77-S100.

60. van het Hof KH, West CE, Weststrate JA, et al. (2000) Dietary factors that affect the bioavailability of carotenoids. J Nutr 130, 503-506.

61. Alberg A (2002) The influence of cigarette smoking on circulating concentrations of antioxidant micronutrients. Toxicology 180, 121-137.

62. Crozier A, Jaganath IB \& Clifford MN (2009) Dietary phenolics: chemistry, bioavailability and effects on health. Nat Prod Rep 26, 1001-1043.

63. Kanaze IF, Bounartzi MI, Georgarakis M, et al. (2006) Pharmacokinteics of the citrus flavanone aglycones hesperetin and naringenin after single oral administration in human subjects. Eur J Clin Nutr 61, 472-477.

64. Cantilena LR, Stukel TA, Greenberg ER, et al. (1992) Diurnal and seasonal variation of five carotenoids measured in human serum. Am J Clin Nutr 55, 659-663.

65. Macdonald HM, Hardcastle AC, Duthie GG, et al. (2009) Changes in vitamin biomarkers during a 2-year intervention trial involving increased fruit and vegetable consumption by free-living volunteers. Br J Nutr 102, 1477-1486. 